\title{
High-Speed High-Efficiency Large-Area Resonant Cavity Enhanced p-i-n Photodiodes for Multimode Fiber Communications
}

\author{
M. Gökkavas, O. Dosunmu, M. S. Ünlü, Senior Member, IEEE, G. Ulu, R. P. Mirin, D. H. Christensen, and E. Özbay
}

\begin{abstract}
In this letter, we report AlGaAs-GaAs p-i-n photodiodes with a 3-dB bandwidth in excess of $10 \mathrm{GHz}$ for devices as large as 60- $\mu \mathrm{m}$ diameter. Resonant cavity enhanced photodetection is employed to improve quantum efficiency, resulting in more than $90 \%$ peak quantum efficiency at $850 \mathrm{~nm}$.
\end{abstract}

Index Terms-High-speed optoelectronics, photodetectors, photodiodes, resonant cavity, pin diodes.

\section{INTRODUCTION}

$\mathbf{V}$ ERTICAL-CAVITY surface-emitting lasers (VCSEL) emitting at $850 \mathrm{~nm}[1]$ have become the preferred source for high-speed short-wavelength communication systems. These VCSELs are particularly suitable for local area networks (LAN) using multimode optical fibers (MMF) with typical core diameters of 50 and $62.5 \mu \mathrm{m}$. While the circular output beam of the VCSELs allow for easy coupling of light into the $\mathrm{MMF}$, it is also desirable to have photodetectors with large active windows compatible with MMF for low-cost coupling of light at the receiving end. Furthermore, high sensitivity and high bandwidth are also necessary attributes for photodetectors used in optical communication applications. As the bandwidth of graded-index multimode fibers continue to improve [2], and VCSELs with modulation rates approaching $10 \mathrm{~Gb} / \mathrm{s}$ become commercially available [3], compatible high-speed performance is required from photodetectors. In this letter, we report high bandwidth $(10 \mathrm{GHz})$ high efficiency $(>90 \%)$ large active area (60 $\mu \mathrm{m}$ diameter) photodiodes operating at $850-\mathrm{nm}$ wavelength.

The two major speed-limiting factors in vertically illuminated photodiodes are the depletion capacitance and the transit time [4]. The capacitance limit can be alleviated either by employing smaller device areas or by increasing the depletion width, thereby decreasing the capacitance per unit area. However, an increased depletion width consequently increases the transit time. Therefore, for any given device area, there is an

Manuscript received May 29, 2001; revised August 24, 2001.This work has been supported in part by the National Science Foundation under Grant no. INT-9 601770 and under Grant ECS-9625236, and by the Army Research Laboratory Cooperative Agreement under DAA17-99-2-0070.

M. Gökkavas, O. Dosunmu, and M. S. Ünlü are with the Department of Electrical and Computer Engineering, Boston, MA 02215 USA.

G. Ulu is with the Department of Physics, Boston, MA 02215 USA.

R. P. Mirin and D. H. Christensen are with the National Institute of Standards and Technology, Boulder, CO 80303 USA.

E. Özbay is with the Department of Physics, Bilkent University, Bilkent, Turkey.

Publisher Item Identifier S 1041-1135(01)09983-9. optimum depletion width that results in the maximum device bandwidth.

Utilization of a thin absorption layer at an optimized position within the depletion region can further improve the transit-time-limited bandwidth of conventional photodiodes. We can compensate for the reduction in quantum efficiency resulting from a thin absorption layer by using resonant cavity enhanced (RCE) detection [5], [6]. In RCE, the active structure is placed in a microcavity, enhancing the optical field at resonant wavelengths, and hence, drastically increasing the quantum efficiency. As RCE is particularly attractive for thin depletion regions, reports of high-speed RCE detectors to date are concerned with small active area (on the order of 10-20 $\mu$ m-diameter windows), transit-time-limited devices [7]-[9]. Conventional photodiodes with 70- $\mu \mathrm{m}$-diameter windows are commercially available, however, their $3-\mathrm{GHz}$ bandwidth [10] is significantly low when compared with the $10-\mathrm{Gb} / \mathrm{s}$ modulation capacity of VCSELs [3]. The present work reports design, fabrication, and characterization of AlGaAs-GaAs RCE p-i-n photodiodes for short distance fiber-optic communications. Large active area $(60 \mu \mathrm{m}$ diameter) devices operating at $850 \mathrm{~nm}$ showed 3-dB bandwidths of $10 \mathrm{GHz}$ with a quantum efficiency of more than $90 \%$.

\section{DEVICE DESIGN AND FABRICATION}

In our large area $\mathrm{p}-\mathrm{i}-\mathrm{n}$ photodiode structure, shown in Fig. 1 , the total depletion width of $1.3 \mu \mathrm{m}$ is chosen so that for a $40-\mu \mathrm{m}$-diameter device the transit-time-limited and capacitance limited bandwidths (for a 50- $\Omega$ load resistance) are equal. Clearly, smaller devices, although not optimized, would yield larger bandwidths, and larger devices would not reach the maximum bandwidth possible for their size. The position of the GaAs absorption region is optimized to minimize the transit time of electrons and holes for the $1.3-\mu \mathrm{m}$-thick depletion region. The estimated transit-time-limited $3-\mathrm{dB}$ bandwidth is 35 GHz. The RC-limited bandwidth is calculated as $12.7 \mathrm{GHz}$ for a $60-\mu \mathrm{m}$-diameter device and linearly scales with inverse of device area.

The design of the epilayer structure shown in Fig. 1 is adjusted to obtain a resonance peak at $850 \mathrm{~nm}$. The layers are grown by molecular beam epitaxy. The AlGaAs-GaAs interfaces are alloy-graded for $30 \mathrm{~nm}$ to eliminate charge trapping that may adversely affect the high-speed performance. At the operating wavelength, only the GaAs region absorbs the optical excitation, as the $\mathrm{Al}$ mole fraction of AlGaAs throughout the 


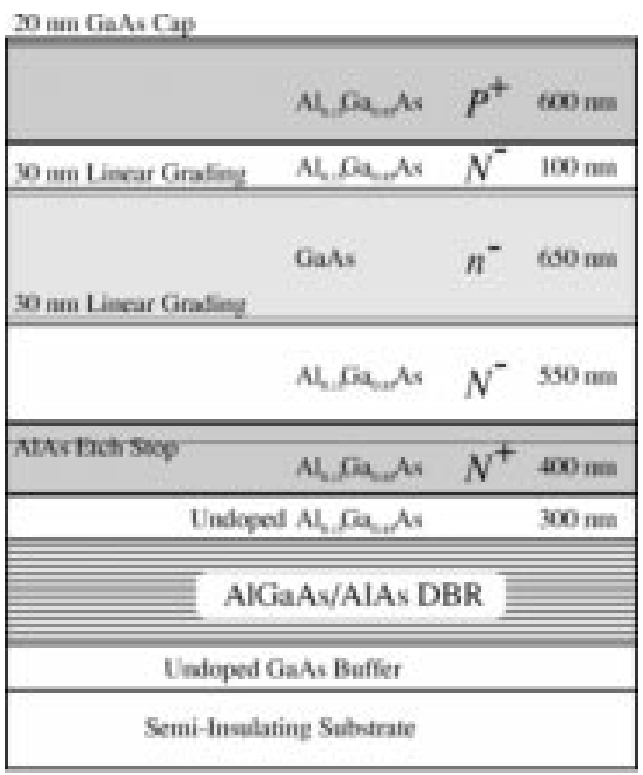

Fig. 1. Schematic representation of the layer structure of the large-area RCE p-i-n photodiodes.

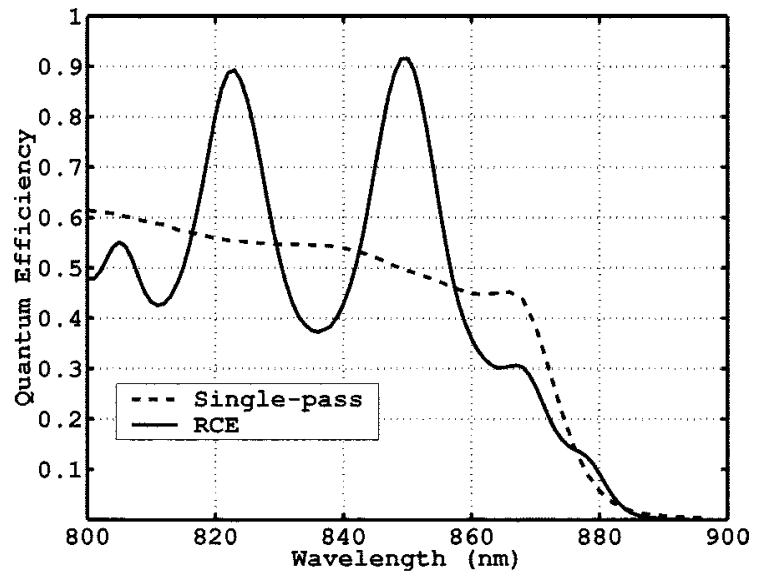

Fig. 2. Measured spectral response of the photodiodes. The solid and dashed curves correspond to the RCE and single-pass control devices, respectively.

cavity and in the DBR is chosen as $15 \%$ to ensure a negligible optical loss. Conventional single-pass control devices were also fabricated on a similar wafer having the exact same layer structure with the exception of the DBR. Both RCE and conventional devices demonstrated breakdown voltages larger than $15 \mathrm{~V}$ and typical dark current values of less than $10^{-6} \mathrm{~A} / \mathrm{cm}^{2}$ at $1 \mathrm{~V}$ reverse bias.

\section{EXPERIMENTAL RESULTS}

For spectral photoresponse characterization, a computer controlled monochromator is used, and the response is calibrated by using a NIST-traceable optical powermeter. Fig. 2 shows the spectral quantum efficiency measurements in the 800-900-nm range at $0-\mathrm{V}$ bias for an $\mathrm{RCE}$ detector and a single-pass control device. A peak quantum efficiency of more than $90 \%$ is measured at the resonance wavelength of $850 \mathrm{~nm}$ for the RCE device, compared to $50 \%$ efficiency for the control device without

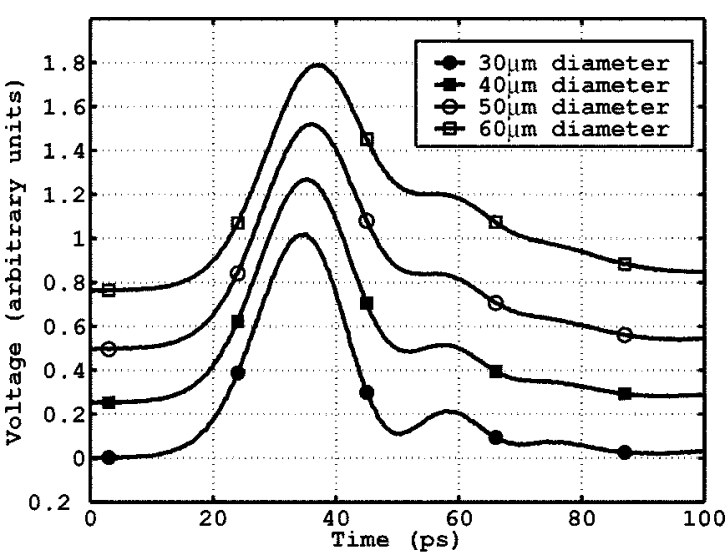

Fig. 3. Temporal response of 30-, 40-, 50-, and 60- $\mu$ m-diameter circular RCE $\mathrm{p}$-i-n photodiodes obtained at $5 \mathrm{~V}$ reverse bias. The results are offset for clarity.

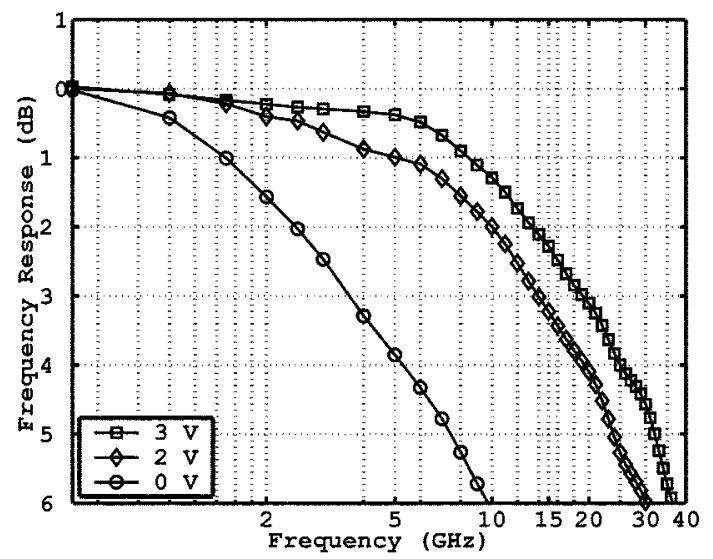

Fig. 4. The FFT of the temporal response measured at $0 \mathrm{~V}, 2 \mathrm{~V}$, and $3 \mathrm{~V}$ dc reverse bias from a $30 \mu \mathrm{m}$-diameter device. There is a dramatic increase in the bandwidth when the reverse bias is increased from $0 \mathrm{~V}$ to $2 \mathrm{~V}$.

RCE. The position of the spectral peaks is adjustable by a surface recess [7].

High-speed measurements are performed on a microwave probe station by using $1.6 \mathrm{ps}$ full-width at half-maximum (FWHM) pulses from a mode-locked Ti:sapphire laser, and the pulse response is observed on a 50-GHz sampling oscilloscope. Fig. 3 shows the temporal response obtained at $5-\mathrm{V}$ reverse bias from 30-, 40-, 50-, and 60- $\mu$ m-diameter circular devices with respective measured FWHM values of 17, 18, 20, and 22 ps. The variation in measured risetime is relatively small, with rise time increasing from $13 \mathrm{ps}$ for $10-\mu \mathrm{m}$-diameter devices to $14 \mathrm{ps}$ for $60-\mu \mathrm{m}$-diameter devices.

The overall bandwidth of the measurement setup is in excess of $30 \mathrm{GHz}$ allowing for accurate measurement of highspeed response of the devices we studied. To determine the frequency response, measured temporal response data are converted to frequency domain using fast Fourier transform (FFT). For each FFT calculation, two sets of raw time-domain data with 2 ns and 500 ps total time spans, each with 4096 samples, are used. Fig. 4 shows the FFT obtained from a $30-\mu \mathrm{m}$ diameter for $0-, 2-$, and $3-\mathrm{V}$ reverse bias. A minimum of $3-\mathrm{V}$ reverse bias is required to fully deplete the $1.3-\mu \mathrm{m}$-thick $n^{-}$region. At larger bias values, diffusion and carrier trapping are eliminated, and only capacitance and transit time limit the response. In the $0-3-\mathrm{V}$ bias range, with increasing bias, the edge 


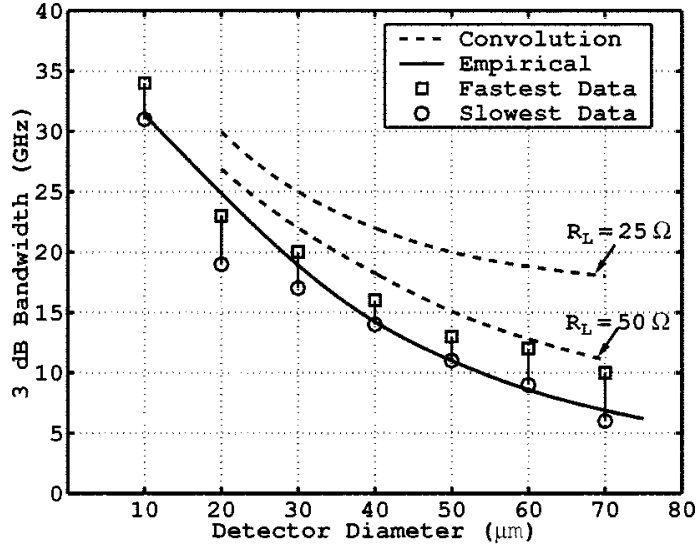

Fig. 5. Measured and calculated 3-dB bandwidth versus detector diameter. For any device size, squares and circles represent experimental variation of the highest and lowest measured bandwidths. The solid and the two dashed curves represent theoretical expectations (See text for a detailed description).

of the depleted region moves further into the $n^{-}$region, decreasing capacitance. Furthermore, at zero bias, the GaAs absorption layer is not fully depleted. The diffusion current due to carriers generated in the undepleted portion of the absorption region, as well as carrier trapping at the graded absorber boundary, result in a long tail that causes the sharp rolloff at low frequencies. In comparison, the difference in the frequency responses measured at 2 and $3 \mathrm{~V}$ is due to the change in capacitance. The 3 - $\mathrm{dB}$ bandwidth determined by FFT from time-domain measurements at $5-\mathrm{V}$ bias, versus detector diameter is plotted in Fig. 5. There was no difference in frequency response between RCE and single-pass devices. The transit-time dominates the response of $10-\mu \mathrm{m}$-diameter devices, whereas the capacitance charging time dominates the response of $50 \mu \mathrm{m}$ diameter and larger devices. The three curves in Fig. 5 represent theoretical expectations. The solid curve is plotted by employing an empirical method, where an overall time constant is calculated as the sum of the RC time constant and transit time [6]. First, the RC time constant (assuming a 50- $\Omega$ load resistance) for each device size is calculated. Then, the $3-\mathrm{dB}$ bandwidth was deduced by weighing the effect of the RC time constant and the transit time ( $\sim 13$ ps), equally. On the other hand, the two dashed curves are obtained by convolving a decaying exponential function (corresponding to the RC time constant with either a 25 - or 50- $\Omega$ load resistance) with the response of a $10-\mu \mathrm{m}$-diameter device, with the consideration that it represents the total system response to a solely transit-time limited device. Although neither of the two methods represents an accurate model, the overall trend is intuitive and matches the experimental results. From Fig. 5, it is seen that the measured 3-dB bandwidth is in excess of $10 \mathrm{GHz}$ for devices as large as $60 \mu \mathrm{m}$ diameter. It should be noted that all $3-\mathrm{dB}$ bandwidths reported here are measured with a $50-\Omega$ load resistance. For larger area devices (50 $\mu \mathrm{m}$ diameter and larger), the response is capacitance limited, and decreasing the load resistance can substantially increase the bandwidth as illustrated by the two dashed curves in Fig. 5. For example, if a $50 \Omega$ parallel load resistor is added, reducing the total load resistance to $25 \Omega$, a $20-\mathrm{GHz}$ bandwidth for a $50-\mu \mathrm{m}$-diameter detector can be achieved. With $25-\Omega$ load resistance, although the overall V/W responsivity of the detector will be reduced by a factor of two, it is still reasonably high (approximately $15 \mathrm{~V} / \mathrm{W}$ ) due to the very high efficiency afforded by the RCE design. ( $\eta>90 \%$, Responsivity $=0.62 \mathrm{~A} / \mathrm{W}$ at $\lambda=850 \mathrm{~nm}$.)

With increasing bit rate, the average receiver power would also increase and photodiodes with high-power handling capability are going to be important. The RCE scheme provides for high-efficiency for the given layer structure, which in turn allows for larger active areas compared to conventional photodiodes with similar high-speed and efficiency performance. As a result, we expect similar or improved saturation behavior compared to conventional top-illuminated photodiodes.

\section{CONCLUSION}

We have demonstrated large active-area AlGaAs-AlAs RCE p-i-n photodiodes with a peak quantum efficiency of $>90 \%$ at $850 \mathrm{~nm}$. The $3-\mathrm{dB}$ bandwidth measured with a $50-\Omega$ load resistance is more than $10 \mathrm{GHz}$ for a circular device with $60 \mu \mathrm{m}$ diameter. The experimentally demonstrated performance of these photodiodes exceeds the detector requirements for $10-\mathrm{Gb} / \mathrm{s}$ optical communication systems. With a factor of two reduction in the load resistance, a bandwidth of more than $20 \mathrm{GHz}$ would be attainable for photodiodes having as large as $50 \mu \mathrm{m}$ diameters with potential for $40-\mathrm{Gb} / \mathrm{s}$ applications. The wavelength selectivity of the RCE structure, which could represent a difficulty in applications, can be overcome by AR coating the top surface. The resulting double-pass detector would yield $75 \%$ quantum efficiency.

\section{ACKNOWLEDGMENT}

The views and conclusions contained in this document are those of the authors and should not be interpreted as representing the official policies, either expressed or implied, of the Army Research Laboratory or the U.S. Government.

\section{REFERENCES}

[1] W. W. Chow, K. D. Choquette, M. H. Crawford, K. L. Lear, and G. R. Hadley, "Design, fabrication, and performance of infrared and visible vertical-cavity surface-emitting lasers," IEEE J. Quantum Electron., vol. 33, pp. 1810-1824, Oct. 1997.

[2] GigaGuide 50 and GigaGuide 62.5 Specification Sheet, Lucent Technologies.

[3] 10 Gbps VCSEL Specification Sheet, Emcore Optical Devices.

[4] J. E. Bowers and C. A. Burrus, "Ultrawide-band long-wavelength $p-i-n$ photodetectors," J. Lightwave Technol., vol. LT-5, pp. 1339-1350, May 1987.

[5] M. S. Ünlü and S. Strite, "Resonant cavity enhanced photonic devices," Appl. Phys. Rev. J. Appl. Phys., vol. 78, no. 2, pp. 607-639, 1995.

[6] M. S. Ünlü, B. M. Onat, and Y. Leblebici, "Transient simulation of heterojunction photodiodes, part II: Analysis of resonant cavity enhanced photodetectors," J. Lightwave Technol., vol. 13, pp. 396-405, Mar. 1995.

[7] E. Özbay, I. Kimukin, N. Biyikli, O. Aytür, M. Gökkavas, G. Ulu, R. P. Mirin, K. A. Bertness, D. H. Christensen, and M. S. Ünlü, "Highspeed $>90 \%$ quantum-efficiency $p-i-n$ photodiodes with a resonance wavelength adjustable in 795-835 nm range," Appl. Phys. Lett., vol. 74, no. 8, pp. 1074-1076, 1999.

[8] B. M. Onat, M. Gökkavas, E. Özbay, E. P. Ata, E. Towe, and M. S. Ünlü, "100 GHz resonant cavity enhanced Schottky photodiodes," IEEE Photon. Technol. Lett., vol. 10, pp. 707-709, May 1998.

[9] C. C. Barron, C. J. Mahon, B. J. Thibeault, G. Wang, W. Jiang, L. A. Coldren, and J. E. Bowers, "Resonant-cavity-enhanced pin photodetector with $17 \mathrm{GHz}$ bandwidth-efficiency product," Electron. Lett., vol. 30, pp. 1796-1797, 1994.

[10] Gigarray Specification Sheet, Emcore Optical Devices. 